\title{
ADAPTIVE FINITE ELEMENT METHODS FOR SHAPE OPTIMIZATION OF LINEARLY ELASTIC STRUCTURES*
}

\author{
Noboru KIKUCHI, Kyoon Yang CHUNG, Toshikazu TORIGAKI \\ and John E. TAYLOR \\ The University of Michigan, Ann Arbor, MI 48109, U.S.A.
}

Received July 1985

\begin{abstract}
Grid adaptive methods combined with means for automatic remeshing are applied to problems in shape optimal design of linearly elastic structures. The quantitative effect of element distortion near the design boundaries is identified in terms of interpolation error associated with the finite element discretization. The grid adaptation is itself formulated as a structural optimization problem, with an objective function that reflects the discretization error. A 'necessary condition' from this formulation provides the basis for a computational procedure to predict the modified grid.

To avoid the sometimes drastic distortion of the FEM grid that might otherwise occur in conjunction with design change, remeshing must be performed at intermediate stages of the overall solution process. In order to produce results for the optimal shape design without interruption in this process, the computer program combines numerical grid generation and automatic remeshing with the grid adaptation and design change. Results for several shape design problems obtained with the use of grid adaptation are compared to computational results predicted from a fixed grid. Both ' $r$-' and ' $h$ adaptation' are tested.
\end{abstract}

\section{Introduction}

Shape design of linearly elastic structures has been studied during this decade after the intensive developments of finite element and optimization methods. Zienkiewicz and Campbell [1] applied finite element and penalty methods for shape optimization problems in 1973. Other studies were reported by Ramakrisnan and Francavilla [2], Tvergaard [3], Kristensen and Madsen [4], Queau and Trompette [5], Oda and Yamazaki [6], and others. Tvergaard applied finite different methods in curvilinear coordinates for the shape optimization of a fillet to minimize the maximum elastic stress for a given load. Queau and Trompette used straight lines and circles to describe the design boundaries instead of applying (piecewise) polynomials to represent arbitrary shape of the boundaries.

Analysis and theory of shape optimization have been given by, e.g., Banichuk [7], Dems [8], Dems and Mroz [9-11], Choi and Haug [12], $\mathrm{Na}$ et al. [13], and others. Dems solved the problem of minimizing the cross-sectional area of a torsion bar constraining the maximum torsional and bending rigidity. Dems and Mroz provided the first variations w.r.t. shape change

\footnotetext{
* The authors' work has been supported partially by NASA Lewis Research Center through the grant NAG3-388. This support is gratefully acknowledged.
} 
of arbitrary stress/strain/displacement functionals, expressed in terms of the stress and strain fields of primary and adjoint structures. On the other hand, Choi and Haug have exploited the idea of the material derivative in continuum mechanics to derive the design sensitivity. They express design change as design deformation velocity in the dynamic process of deformation of continuum. By employing the material derivative of the variational equation and replacing the material derivative of the response by the virtual displacement they introduce an adjoint equation which leads to design sensitivity from the performance functional. $\mathrm{Na}$ et al. applied the idea of structural remodeling introduced by Olhoff and Taylor [14] to solve shape optimization problems for a tension bar.

The underlying goal of the development effort reported in this paper was to establish dependable means for the automatic computational determination of optimal shape design. The basis from analytical modeling for the performance of shape design is generally available, at least for linearly elastic, small-deformation continuum problems. However, certain difficulties that were encountered in efforts to implement results from analysis in methods for computational solutions had remained unresolved. We refer to the kinds of difficulties that manifest themselves in the form of instabilities in the computational procedures, convergence to inappropriate shape designs, and so on (Fig. 1). Also, it appears that, compared to the usual computational analysis for structures, computational schemes for shape design are more sensitive to the approximation error associated with discretization modeling.

As the shape of a structure changes in the process of computation for shape design, the associated mesh for a finite element method (FEM) model of the system may become distorted. Because the prediction of shape change depends on the quality of results from application of the FEM, excessive distortion and the consequent degradation of these results must be controlled. We incorporate a device for automatic mesh regeneration into the overall computational procedure as a means to achieve this control. At the same time, error inherent in the FEM approximation, i.e., error associated with grid size, interpolation forms, etc., may result in poor
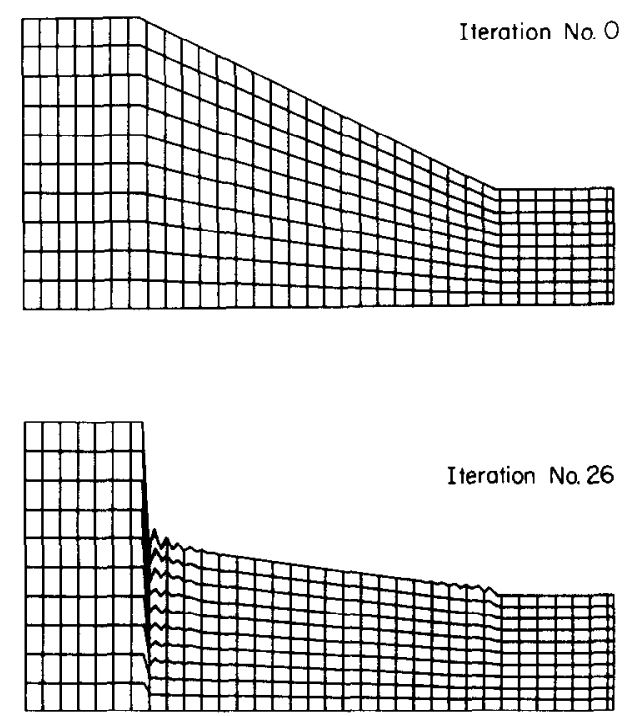

Fig. 1. The initial and optimum shapes and finite element grids without remeshing. 
performance of procedures for shape design. Where an improvement in the quality of the approximation is needed, steps for the adaptive refinement of the FEM model itself are introduced.

The need to have grid adaptation with shape optimization problems was recognized in the treatment of the fillet design problem, for example. Adaptive grid design introduced in Kikuchi [15] is combined with automatic remeshing and adaptive finite element methods such as those studied extensively by Babuška and his coworkers [16-20], Shephard [21], Zienkiewicz et al. [22], and others. Details of adaptive finite element methods and related literature can be found in [23-25]. In the present treatment, the scheme for mesh refinement operates in a way to produce the optimum adaptation of the FEM grid.

\section{Formulation for shape optimal design}

We follow the development reported by Chung [26] to consider shape design problems where the criterion for optimization is the maximum value over the design domain of a local measure of performance. Depending on the practical requirements for a design, the local measure might reflect magnitude of displacement, von Mises stress, or maximum component of shear stress, for example. With the local measure expressed in general form as function $F(u, \nabla u)$, where $u$ symbolizes the displacement (state) vector, the design problem can be stated as:

$$
\begin{array}{ll}
\operatorname{Min}_{\Gamma_{\mathrm{d}}} & \left\{\operatorname{Max}_{x \in \Omega}|F(u, \nabla u)|\right\}, \\
\text { s.t. } & \int_{\Omega} \mathrm{d} \Omega-A \leqslant 0 .
\end{array}
$$

$\Gamma_{\mathrm{d}}$ and $\Omega$ represent the design boundaries of the structure and its domain. Admissible designs are required to lie within the stated isoperimetric constraint on $\Omega$, where the bound $A$ is specified. Also it is understood that vector $u$ satisfies equilibrium (state) equations and associated boundary conditions.

With the introduction of an additional unknown $\beta$, the design problem can be expressed as (equivalence of the problem statements is substantiated in [28]):

$$
\begin{array}{ll}
\operatorname{Min}_{\Gamma_{\mathrm{d}}} & \beta, \\
\text { s.t. } & \int_{\Omega} \mathrm{d} \Omega-A \leqslant 0, \\
& |F(u, \nabla u)|-\beta \leqslant 0 \text { in } \Omega .
\end{array}
$$

The Lagrangian for this scalar minimization problem,

$$
\begin{aligned}
L(u, v, \Lambda, \lambda, \beta)= & \beta+\int_{\Omega}\left[\Lambda_{1}\left\{\sigma_{i j}(u) e_{i j}(v)-b_{i} v_{i}\right\}+\lambda_{1}\{F(u, \nabla u)-\beta\}\right. \\
& \left.-\lambda_{2}\{F(u, \nabla u)+\beta\}+\Lambda_{2}\right] \mathrm{d} \Omega-\int_{\Gamma_{i}} \Lambda_{1} t_{i} v_{i} \mathrm{~d} \Gamma-\Lambda_{2} A,
\end{aligned}
$$


is written to reflect explicitly the equilibrium constraint on $u$, expressed here via the virtual displacement equations for two-dimensional elasticity problems. Traction $t$ is specified on $\Gamma_{t}$ and portions $\Gamma_{u}$ and $\Gamma_{0}$ of the boundary are 'fixed' and 'free' respectively. The usual conditions for 'virtual displacement' serve to define the set of admissible functions.

A partial listing of necessary conditions for this problem follows (for simplicity the equations are written for the case $\lambda_{2} \equiv 0$ ):

$$
\begin{aligned}
& 1-\int_{\Omega} \lambda_{1} \mathrm{~d} \Omega=0 \quad \text { in } \Omega, \\
& \Lambda_{1} \sigma_{i j}(v)_{, j}=\lambda_{1}\left(\partial F / \partial u_{j}\right)-\left[\lambda_{1}\left(\partial F / \partial u_{i, j}\right)\right]_{, j} \quad \text { in } \Omega, \\
& v=0 \quad \text { on } \Gamma_{u}, \\
& \sigma_{i j}(v) n_{j}=\lambda_{1}\left(\partial F / \partial u_{i, j}\right) n_{j} \quad \text { on } \Gamma_{0} \text { and } \Gamma_{t}, \\
& \Lambda_{1}\left[\sigma_{i j}(u) e_{i j}(v)-b_{i} v_{i}\right]+\lambda_{1}(F-\beta)=-\Lambda_{2} \text { on } \Gamma_{\mathrm{d}}, \\
& \lambda_{1}(F-\beta)=0, \quad \lambda_{1} \geqslant 0, \quad F-\beta \leqslant 0 \quad \text { in } \Omega .
\end{aligned}
$$

Equation (6) reflects a normalization on multiplier $\lambda_{1}$ (or more generally on $\lambda_{1}$ and $\lambda_{2}$ ) associated with the criterion constraint (4), which appears as a 'load' in the 'adjoint equilibrium problem' (7). Where $\lambda_{1}$ is singular, the singularities are identified via (6) as Dirac functions. Equation (8) is simplified using (9) to obtain the 'optimality condition'

$$
\sigma_{i j}(u) \varepsilon_{i j}(v)-b_{i} v_{i}=-\Lambda_{2} / \Lambda_{1} \text { on } \Gamma_{\mathrm{d}} .
$$

Thus, for problems without geometric constraint on the design boundary and with zero body force $b_{i}$, unit mutual energy $\sigma_{i j}(u) \varepsilon_{i j}(v)$ has constant value over the optimally shaped boundary. A more detailed treatment of the reduction of the set of first-order conditions is furnished in [26]. Equivalence between the design problem of (1) and the problem for 'minimization of area $\int_{\Omega} \mathrm{d} \Omega$ within prescribed bound $\beta$ on criterion function $F(u, \nabla u)$ is discussed there as well.

A related but distinct form of design problem is obtained if the minimization applies w.r.t. the design boundary alone rather than w.r.t. to the (closed) domain of the structure. Examples are:

and

$$
\begin{array}{ll}
\operatorname{Min}_{\Gamma_{\mathrm{d}}} & \text { (Area), } \\
\text { s.t. } & |F(u, \nabla u)|-\beta \leqslant 0 \quad \text { on } \Gamma_{\mathrm{d}}
\end{array}
$$

$$
\begin{array}{ll}
\operatorname{Min}_{\Gamma_{\mathrm{d}}} & \left\{\operatorname{Max}_{x \in \Gamma_{\mathrm{d}}}|F(u, \nabla u)|\right\}, \\
\text { s.t. } & \int_{\Omega} \mathrm{d} \Omega-A \leqslant 0 .
\end{array}
$$

Optimality conditions similar to those of (6)-(9) are obtained for these problems, but the 


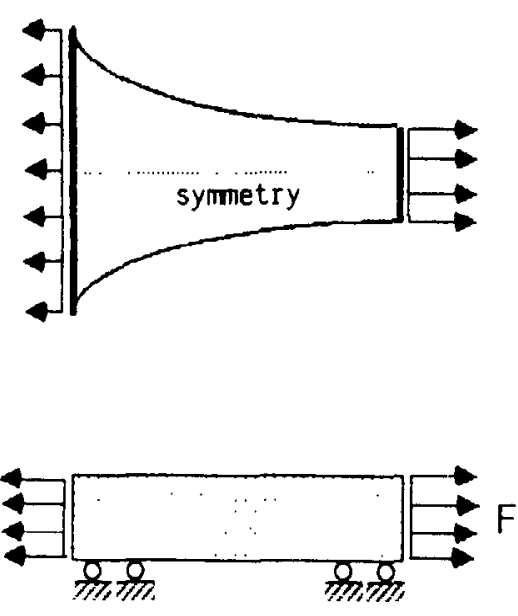

(a)
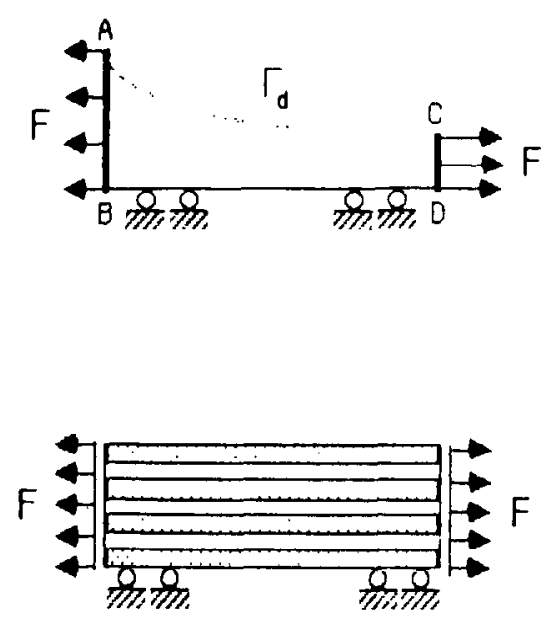

(b)

Fig. 2. Fillet design problem that yields trivial solutions: (a) continuous trivial design; (b) fiber-type trivial design.

adjoint equations and Kuhn-Tucker conditions are different. Equation (12) was used in the work by Francavilla et al. [2] as the approximate formulation of the minimization of stress concentration in domain $\Omega$.

Equation (6) of the necessary conditions for design problem (1) suggests that there exists a measurable set $\Omega_{s} \subset \Omega$ such that $\Omega_{s}=\left\{x \in \Omega: \lambda_{1}(x)>0\right\}$, since the function $F$ is a function in $L^{2}(\Omega)$ for a sufficiently smooth stress field. Then, the local measure $F$ must be saturated in $\Omega_{s}$, That is, $F-\beta=0$ in $\Omega_{s}$. A question is whether $\Omega_{s}$ can be identified with $\Omega$ for a sufficiently 'large' design boundary $\Gamma_{\mathrm{d}}$. As far as our experience is concerned, if $\Omega_{s}$ coincides with $\Omega$, the final designs are sometimes trivial. For example, for the fillet problem shown in Fig. 2, possible solutions are sets of fibers! This suggests that the design boundary $\Gamma_{\mathrm{d}}$ should be appropriately restricted. Another possibility is that the maximum value of the function $F$ in the whole domain can never be reduced by changing the shape of boundaries $\Gamma_{\mathrm{d}}$, if $\Gamma_{\mathrm{d}}$ is overly restricted. For example, if a flat elliptic hole exists inside a given domain $\Omega$ but away from the design boundary, the maximum stress remains located around the hole no matter how the shape of the remote boundaries is changed. Evidently the optimum shape design problem defined in (1) would not be meaningful in such situations. On the other hand, in general, if the maximum of $F$ in $\Omega$ occurs in the neighborhood of the design boundary $\Gamma_{\mathrm{d}}$, shape change implies reduction of the maximum value of $F$. In such cases the design problem according to statements (11) or (12) is practical, while the extension of problem (1) to accommodate constraints on $\Gamma_{\mathrm{d}}$ may not be applicable.

\section{Computational algorithm}

In the treatment of problems for their solution, it is necessary to introduce an interpretation such as those of finite element methods to model the state equation. In the present study we shall apply a finite element approximation based on the displacement method for plane problems using four-node quadrilateral isoparametric elements, in which each component of the 
displacement vector is approximated by a bilinear polynomial. Since, with this approach, only the displacement vector is predicted directly, the stress tensor is computed a posteriori as required.

As in the case of other free boundary problems, shape design problems of linearly elastic structures have been solved using geometric adaptive methods. In most cases, the idea of geometric adaptive methods is stated in the form of a two-step iteration algorithm for the purpose of satisfying the optimality conditions. The first step represents the calculation of some quantities under the assumption that the design boundaries are fixed, and the second step predicts the movement of nodes on the design boundaries in the ratio of differences between the calculated quantities and the given or assumed constants at the nodes of a finite element model.

Optimality conditions of the design problem (1) given in (6)-(9) require constant mutual energy on the design boundary $\Gamma_{\mathrm{d}}$ and the saturation of the design function $F$ in $\Omega_{s}$, i.e., $F-\beta=0$, for the optimal design. If $\Gamma_{\mathrm{d}}$ is contained in the closure of $\Omega_{s}$, both the mutual energy and the design function $F$ become constant on the design boundary $\Gamma_{\mathrm{d}}$. We shall develop a computational algorithm of geometric adaptive methods for shape optimization so that design boundaries are moved in the direction of satisfying these conditions until convergence to the final shape is reached within a certain tolerance.

The expression of the iterative scheme to find new coordinates of nodes on the design boundaries is

$$
x_{i}^{k+1}=x_{i}^{k}+\Delta x_{i}^{k}, \quad i=1, \ldots, N,
$$

for the $k$ th iteration, where $N$ is the total number of nodes on the design boundary. Here, $\Delta x_{i}^{k}$ is the movement of nodes in the specified direction and this value can be obtained from the movement in the normal direction:

$$
\Delta x_{i}=\Delta x_{n i} / \cos \theta_{i}
$$

in the $k$ th iteration. $\theta_{i}$ is the angle between unit normal vector $n_{i}$ and unit 'direction vector' $\alpha_{i}$ at the $i$ th node in the specified direction (Fig. 3). For simplicity, iteration superscript $k$ is omitted in (14) and in the following. The normal movement $x_{n i}$ at the $i$ th node in the $k$ th iteration is obtained from

$$
\Delta x_{n i}=\Delta A_{i} / S_{i}, \quad S_{i}=\frac{1}{2}\left[l_{i-1}+l_{i}\right],
$$

where $\Delta A_{i}$ is the area allotted at the $i$ th node and $l_{i}$ is the length of the $i$ th element on the boundary. The allotted area $\Delta A_{i}$ at the $i$ th node is obtained from the ratio between the stresses $F(\sigma)$ calculated in the previous step of the $k$ th iteration and the prescribed constant $\beta$. Note that the value $\beta$ in $\operatorname{Min}(\operatorname{Max} F)$ can be assumed as the average of $F(\sigma)$ values on the nodes along the design boundary.

$$
\Delta A=\Delta \text { Area }\left[\frac{\left[\left(F_{i}-\beta\right) / \beta\right] S_{i}}{\sum_{i=1}^{N}\left|\left(F_{i}-\beta\right) / \beta\right| S_{i}}\right] .
$$

$F_{i}$ is the value $F(\sigma)$ at node $i$, which is extrapolated from the Gaussian points using least square 


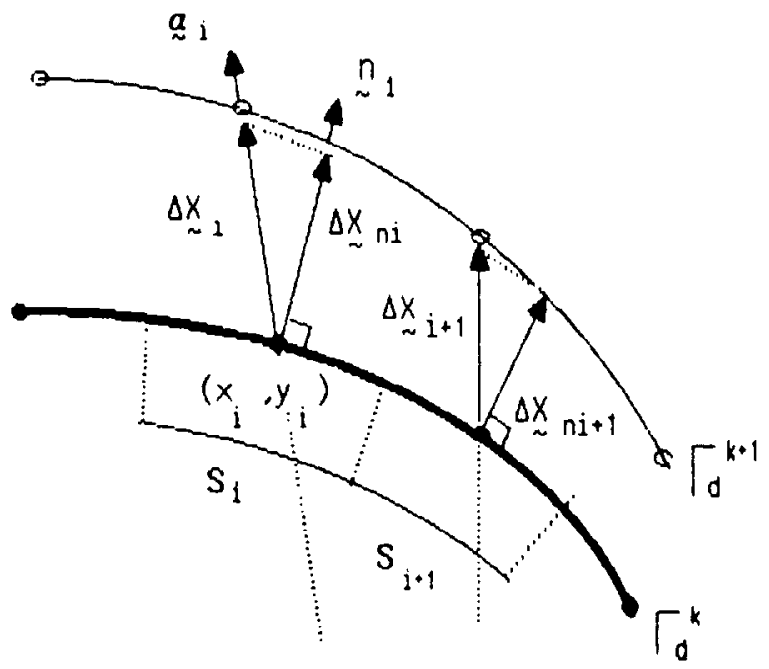

Fig. 3. Geometric adaptive method.

methods, and $\Delta$ Area is the area between the design boundary $\Gamma_{\mathrm{d}}^{k+1}$ and $\Gamma_{\mathrm{d}}^{k}$. The value of $\Delta A$ rea must be large enough at the beginning and diminish as the iteration goes on in order to have convergence. For this purpose we define a percent deviation from the optimum using an $L^{2}$-norm:

$$
\mathrm{DTP}=\left[\frac{1}{N} \sum_{i=1}^{N}\left(\frac{S_{i}\left(F_{i}-\beta\right)}{\beta}\right)^{2}\right]^{1 / 2} \frac{1}{\bar{S}} .
$$

Here $\bar{S}$ is the average length of elements of the design boundary,

$$
\bar{S}=\frac{1}{N-1}\left[\sum_{i=1}^{N} S_{i}\right]
$$

The value of $\triangle$ Area is given by

$$
\Delta \text { Area }=(\text { Total Area of Domain }) \times C \times \text { DTP }
$$

From our experience, the value of $C$ is between zero and 1.0, although the proper value must be adjusted according to the problem and the speed of convergence. That is, the bigger the $C$-value is, the faster the converging speed is. However, faster convergence is sometimes accompanied by oscillations of the design boundaries, and possibly by oscillations of the percent deviation as well, as the iterations proceed. On the other hand, a small value of $C$ may result in very slow convergence without oscillations.

The above algorithm possesses the property that if $F_{i}-\beta \leqslant 0$ is satisfied at a point $x \in \Gamma_{\mathrm{d}}^{k}$, that is at node $i$, then $x$ moves in the direction such that the area $A$ of the domain $\Omega$ is reduced. This may guarantee that, although we have used only the necessary condition, the iteration algorithm leads to the minimum area design automatically. 
One disadvantage of this geometric adaptive method is that the finite element grid can become distorted during the adaptation process. Excessive distortion of finite elements may lead to significant approximation error and a consequent loss of quality in the prediction of shape of design boundaries.

More precisely, if four-node quadrilateral elements are applied for isotropic linearly elastic structures, the components of the strain tensor are approximated by

$$
\varepsilon_{\xi}=A_{1}+C_{1} \eta, \quad \varepsilon_{\eta}=A_{2}+B_{1} \xi, \quad \gamma_{\varepsilon n}=A_{3}+B_{2} \xi+C_{2} \eta,
$$

in the normalized coordinate system $(\xi, \eta)$. This means that, for example, the normal strain in the $\xi$-direction is constant in $\xi$, but is linear in $\eta$. This property may result in a poor approximation in the case that the element distribution is inconsistent with the pattern of the net of equistrain lines as shown in Fig. 4.

To quantify the error in finite element approximations, let us obtain the interpolation error of a function $w$ approximated by four-node finite elements. Suppose that the second derivatives of all the components of the true displacement $u$ are constant in each finite element. Their values need not be the same in different elements. Define

$$
\begin{array}{ll}
A=\partial^{2} w / \partial x^{2}, \quad B=\partial^{2} w / \partial x \partial y, & C=\partial^{2} w / \partial y^{2}, \\
x^{\mathrm{t}}=\left\{x_{1}, x_{2}, x_{3}, x_{4}\right\}, & y^{\mathrm{t}}=\left\{y_{1}, y_{2}, y_{3}, y_{4}\right\}, \\
X^{\mathrm{t}}=\left\{X_{1}, X_{2}, X_{3}, X_{4}\right\}, & Y^{\mathrm{t}}=\left\{Y_{1}, Y_{2}, Y_{3}, Y_{4}\right\}, \\
L_{s}^{\mathrm{t}}=\frac{1}{4}\{-1,1,1,-1\}, & L_{t}^{\mathrm{t}}=\frac{1}{4}\{-1,-1,1,1\}, \\
h^{\mathrm{t}}=\frac{1}{4}\{1,-1,1,-1\}, & \\
J_{11}=\left(L_{s} \cdot x\right)+(h \cdot x) \eta, & J_{12}=\left(L_{s} \cdot y\right)+(h \cdot y) \eta, \\
J_{21}=\left(L_{t} \cdot x\right)+(h \cdot x) \xi, & J_{22}=\left(L_{t} \cdot y\right)+(h \cdot y) \xi, \\
J=J_{11} J_{22}-J_{12} J_{21}, &
\end{array}
$$
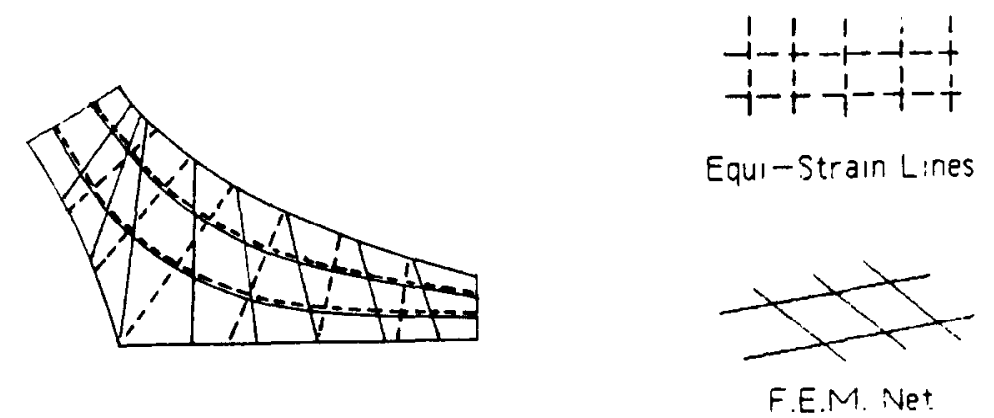

Fig. 4. Conflict between the grid direction and the strain field in a design process. 
where $\left(x_{\alpha}, y_{\alpha}\right)$ are the nodal coordinates of the four corner nodes of a quadratic element in the physical coordinate system, and $\left(X_{\alpha}, Y_{\alpha}\right)$ are four nodes inside of the element, say, the four nodes corresponding to the $2 \times 2$ Gaussian integration points in the master element. It is noted that, if an element is a parallelogram, the terms $h \cdot x$ and $h \cdot y$ become identically zero. Under the assumption stated above, the first derivatives of the difference of the function $w$ and its interpolation $w_{h}$ by the four-node quadrilateral element can be written by

$$
\begin{aligned}
\partial\left(w_{h}-w\right) / \partial x= & (1 / J)\left[-J_{22}\left(J_{11}^{2} A+2 J_{11} J_{12} B+J_{12}^{2} C\right) \xi\right. \\
& +J_{12}\left(J_{21}^{2} A+2 J_{21} J_{22} B+J_{22}^{2} C\right) \eta+\left\{\left(1-\eta^{2}\right) L_{t} y\left(L_{t}-\xi h\right)\right. \\
& \left.+\left(1-\xi^{2}\right) L_{s} y\left(-L_{s}+\eta h\right)\right\} \cdot\{h \cdot x(A X+B Y)+h \cdot y(B X+C Y)\} \\
& +\left\{\xi\left(1-\eta^{2}\right)\left(L_{t}-\xi h\right)+\eta\left(1-\xi^{2}\right)\left(-L_{s}+\eta h\right)\right\} \\
& +\{h \cdot x h \cdot y(A X+B Y)+h \cdot y h \cdot y(B X+C Y)\}] \\
\partial\left(w_{h}-w\right) / \partial y= & (1 / J)\left[-J_{21}\left(J_{11}^{2} A+2 J_{11} J_{12} B+J_{12}^{2} C\right) \xi\right. \\
& +J_{11}\left(J_{11}^{2} A+2 J_{21} J_{22} B+J_{22}^{2} C\right) \eta+\left\{\left(1-\eta^{2}\right) L_{t} \cdot x\left(-L_{t}-\xi h\right)\right. \\
& \left.+\left(1-\xi^{2}\right) L_{s} \cdot x\left(L_{s}+\eta h\right)\right\} \cdot\{h \cdot x(A X+B Y)+h \cdot y(B X+C Y)\} \\
& +\left\{\xi\left(1-\eta^{2}\right)\left(-L_{t}-\xi h\right)+\eta\left(1-\xi^{2}\right)\left(L_{s}+\eta h\right)\right\} \\
& +\{h \cdot x h \cdot x(A X+B Y)+h \cdot x h \cdot y(B X+C Y)\}]
\end{aligned}
$$

where $(\xi, \eta)$ are the normalized coordinates in the master element. This means that if the second derivatives of the solution are known, the interpolation error can be expressed explicitly in terms of $(\xi, \eta)$. Thus the error measure defined above can be computed. If an element is a parallelogram, then the interpolation error becomes very simple, since $h \cdot x=0$ and $h \cdot y=0$, that is, the last two lines are identically zero in each partial derivative. On the other hand, if an element is considerably distorted from a parallelogram, then the terms in the second and third lines in the interpolation error become large in the region where large strain is expected, since $(A X+B Y)$ and other similar terms are basically strain components in an element. This suggests that regular refined finite elements must be set up in the neighborhood of singular points. Here regularity means that an element is close to a rectangle or parallelogram. Otherwise, the error contribution from the terms in the second and third lines would become quite large. This means that grids generated by conformal mappings are very appropriate. Similarly, grids by the elliptic differential equations methods with the orthogonality condition and by the algebraic integer method are suitable in the sense that contribution from the grid distortion and high strain (i.e., stress) can be restricted to be sufficiently small.

Figure 5 shows distribution and amount of error for an example linear elasticity problem. Some finite elements are deliberately distorted in order to see the effect of distortion.

It has been clear that undesirable grid distortion generates unnecessary finite element approximation error. What is an effect of this in shape optimization? An effect can be seen in the example of shape optimization of a triangular plate shown in Fig. 6. If shape optimization is performed using finite element grids shown in Fig. 7, different final shapes are obtained by using 

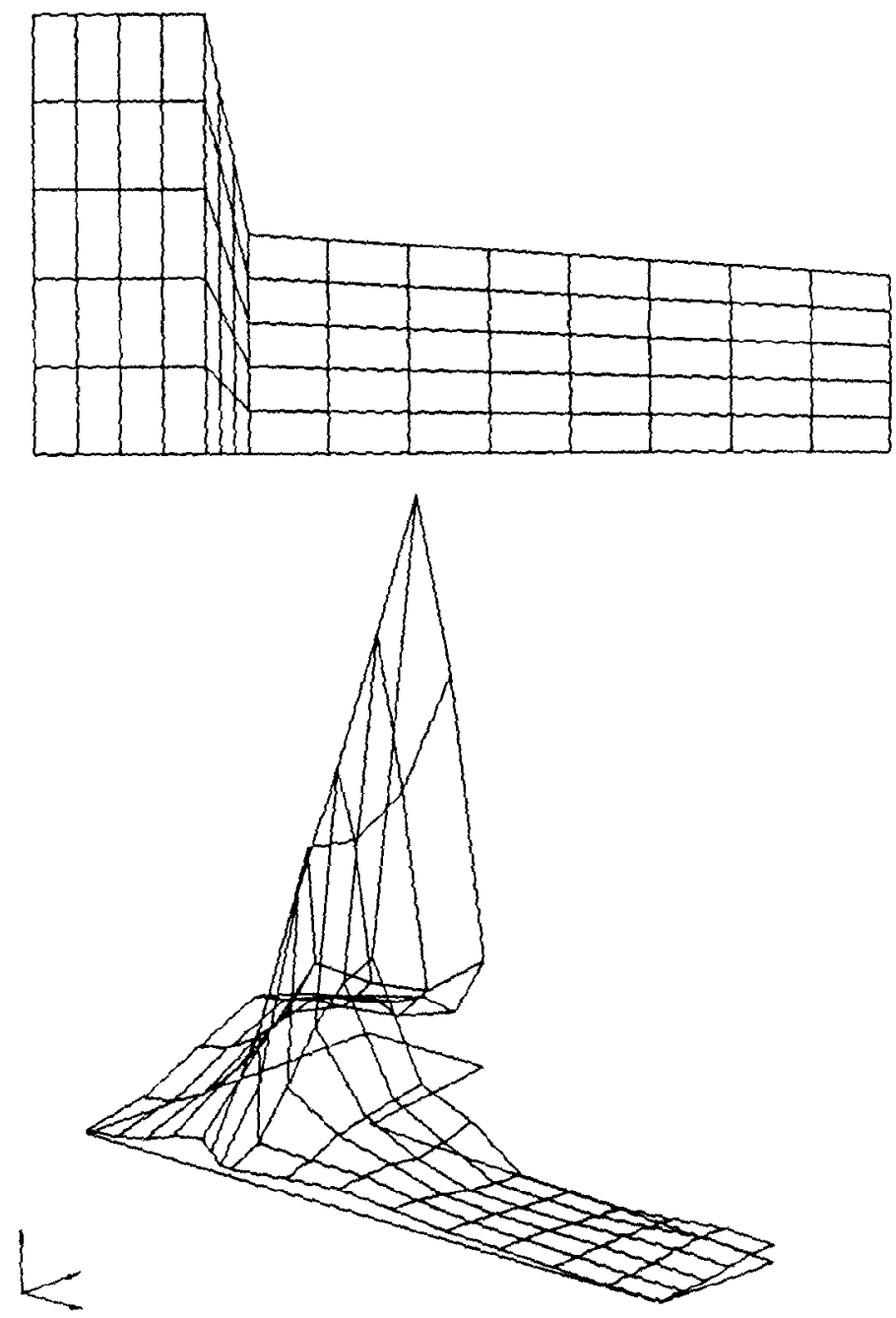

Fig. 5. Distribution of the interpolation error estimated by using form (21).
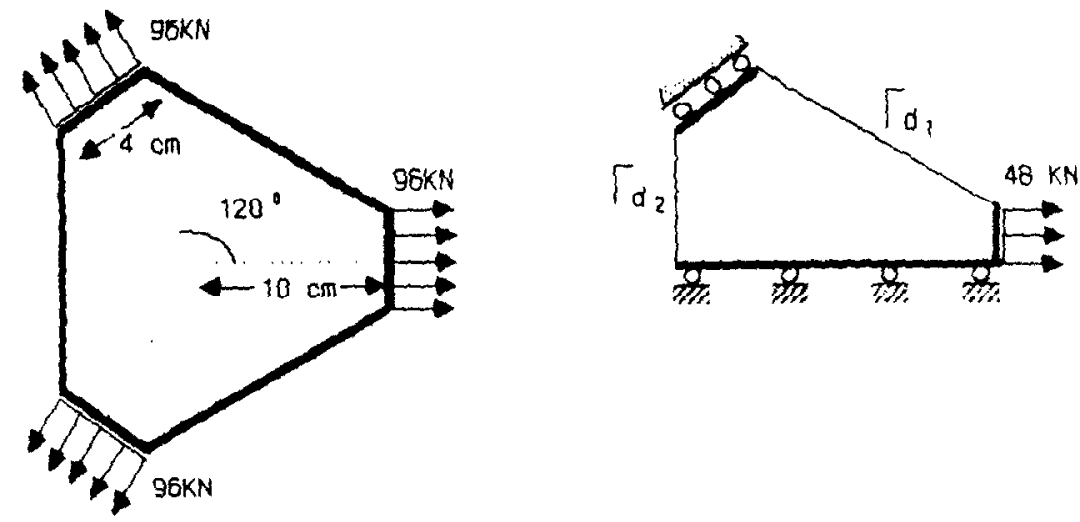

Fig. 6. Shape design problem for a triangular plate. 

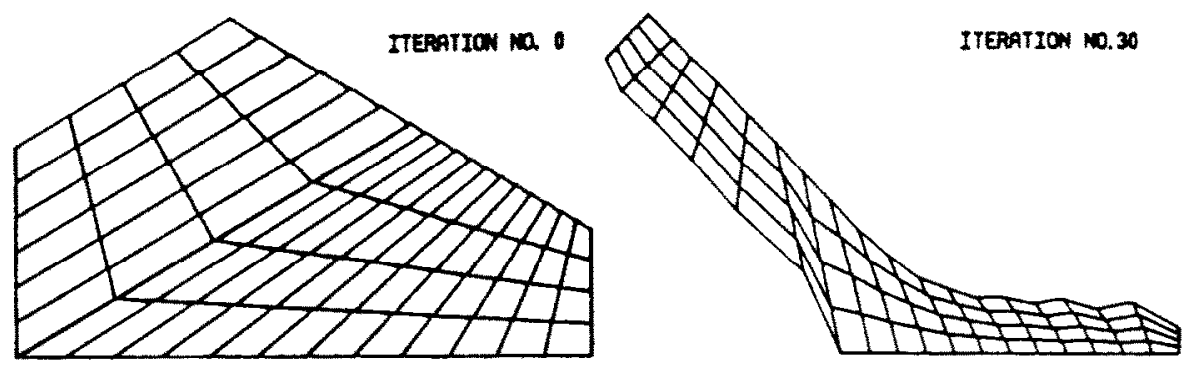

(a)
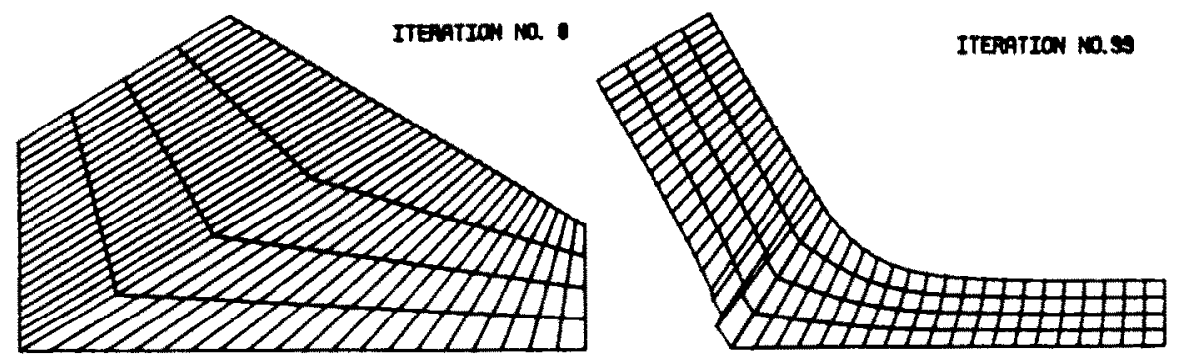

(b)
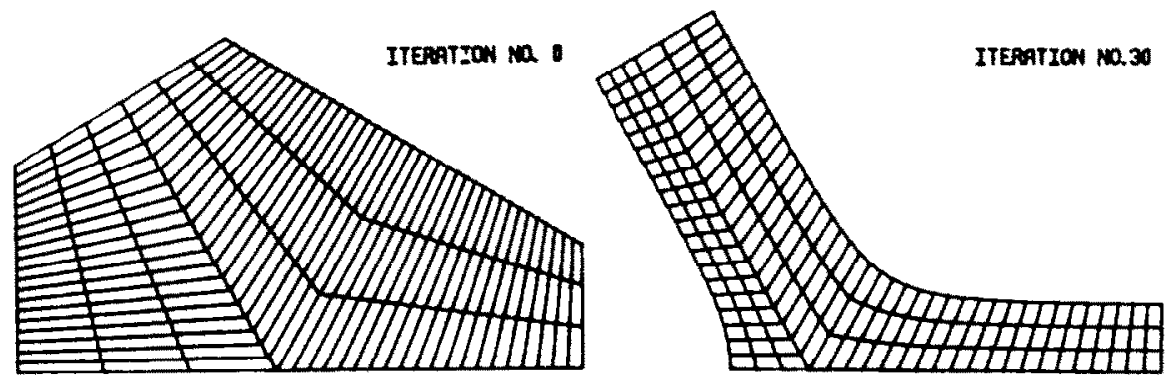

(c)

Fig. 7. Three different optimal shapes obtained using different finite element grids.

different grids. It is clear that the 'regular" grid shown in Fig. 7 (c) yields the smoothest shape to the structure. Then, how can we maintain 'good' grids in the process of shape optimization? One approach is to make us of adaptive finite element methods.

\section{Mesh adaptation and regeneration}

A part of the difficulty that may arise with the computational prediction of shape, namely the development of irregular or nonsmooth boundaries, is moderated with the use of smoothing 
parametric representation (e.g., splines) for the design boundary. However, the commitment to such a representation results in a loss of capability for resolution, i.e., it becomes impossible to simulate intricacies of shape. Also, the use of splines tends to obscure the effect of mesh distortion on the prediction of shape. For these reasons we prefer simply to identify shape with the positions of boundary nodes of the FEM mesh, as indicated above.

Defects in the prediction of shape that might result from error in FEM evaluations because of grid distortion can be limited by including steps for automatic mesh regeneration within the computational process. The critcrion uscd to trigger mesh regeneration is based on the error measure given in (21). Where results from FEM computation lack sufficient quality for reasons other than mesh distortion, mesh adaptation is applied to achieve the required improvement in precision. The mesh refinement used here is itself modelled as an optimal design problem. Proper use of mesh adaptation generally resolves the difficulties with the irregular shape design cited at the start of this section. Also, mesh refinement provides a means to gain proper fidelity for design curves in the neighborhood of sharp variation in the value of state variables (e.g., stress). The design of boundaries near geometric constraints, or around intervals where the boundary is nonconvex, tends to be sensitive in this respect.

A version of the mesh adaptation problem and its application in shape design are summarized in what follows (adaptive FEM has been developed by Babuška and his coworkers, and by others; the reader is referred to the literature for details). We refer to mesh design as 'grid adaptive methods', to distinguish them from geometric adaptation for shape design. Although the ' $p$-method' (the increase in degree of interpolation polynomials) is widely used in finite element adaptation, we shall apply only the ' $r$-method' and ' $h$-method' in this work.

The $r$-method is based on the following formulation for the design of finite element grids. Defining a finite element approximation error in terms of element error measure $E_{e}$, we consider the problem
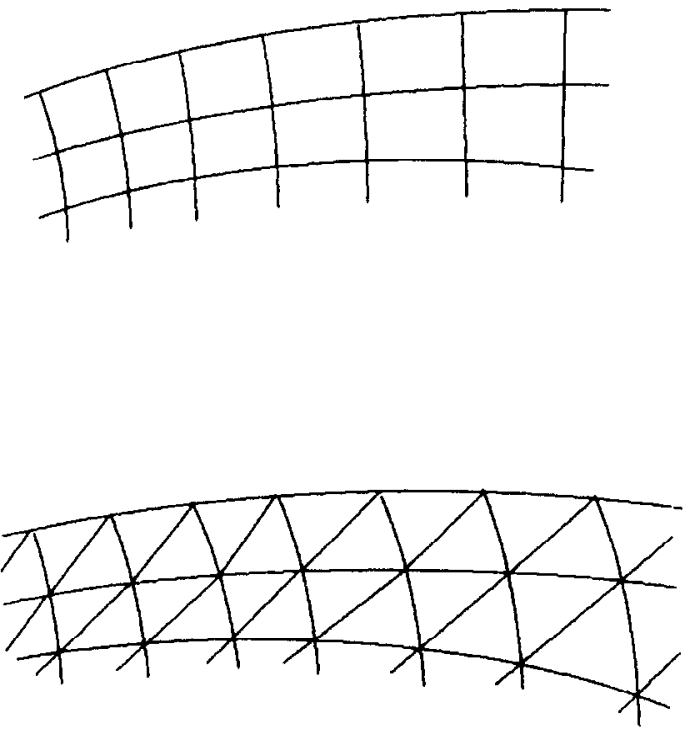

Fig. 8. 'Regular' finite element grids. 


$$
\underset{\text { Node relocation }}{\operatorname{Min}}\left\{\underset{e=1, \ldots, \mathrm{NE}}{\operatorname{Max}} E_{e}\right\} .
$$

Thus the design variables are simply the nodal coordinates. A 'necessary condition' for problem (22) is (see [15], for example)

$$
E_{e}=\text { constant for } e=1, \ldots, \mathrm{NE} .
$$

We proceed to identify an appropriate form for the measure $E_{e}$, and to construct an algorithm for the computational determination of node relocation to satisfy condition (23).

Noting that the finite element approximation error is always bounded by the interpolation error in the displacement method for elliptic boundary value problems, we may take the interpolation error in each element $\Omega_{e}$ as an error measure for the grid design problem, that is,

$$
E_{e}=\sqrt{\int_{\Omega_{e}} E_{i j k l} \varepsilon_{k l}\left(u-v_{h}\right) \varepsilon_{i j}\left(u-v_{h}\right) \mathrm{d} \Omega},
$$

where $E$ are the elasticity constants and $\varepsilon$ is the engineering strain tensor. The first derivatives of the difference $u-v_{h}$ between the solution and its interpolation by four-node elements can be computed as in (21), under the assumption that the second derivatives of the solution are constant in each finite element. Thus the evaluation of the error measure is possible whenever a method to approximate the second derivatives of the solution is given. This can be accomplished in general by applying the least square method to compute continuous strain tensor at nodes in the finite element model. A cruder error measure may be intrdoduced by defining

$$
E_{e}=\sqrt{\int_{\Omega_{e}} F\left(u-v_{h}, \nabla\left(u-v_{h}\right)\right)^{2} \mathrm{~d} \Omega},
$$

using the criterion function $F$ that was introduced for the shape optimization problem. In this case, we do not have an explicit relation for the finite element approximation error. As far as our computational experience is concerned, even a measure such as

$$
E_{e}=\sqrt{\int_{\Omega_{e}}\left(F-F_{h}\right)^{2} \mathrm{~d} \Omega}
$$

works well for certain problems, where $F_{h}$ is the interpolation of $F$ by four-node finite elements. Here also the error measure does not have any direct relationship to the finite element error in mathematics.

The method of relocation of nodal points applied in this work is based on Winslow [27]. The new location of the $n$th node is defined by

$$
x_{n}=\sum_{e} x_{e}^{\mathrm{c}}\left(E_{e} / A_{e}\right) / \sum_{e}\left(E_{e} / A_{e}\right),
$$

where the summation is taken over the finite elements connecting to the $n$th node, $x_{e}^{\mathrm{c}}$ is the coordinate of the centroid of $\Omega_{e}$, and $A_{e}$ is its area. This node relocation scheme does not relocate nodes if error indicators satisfy the necessary condition for a finite element grid 
consisting of rectangular elements. Furthermore, if finite elements are distorted (not because of the solution characteristics), the relocation scheme tends to enforce grids to be 'regular' so that they are close to uniform grids (Fig. 8). Another property of (27) is that refined finite elements are automatically assigned near the vertices of nonconvex corner points of a polygon, since (27) is a very crude difference approximation of the Laplace equation for grid generation. Therefore, (27) is not only useful for enforcement of the necessary condition of optimality to the grid design problem, but also for maintaining regularity of finite element grids with respect to the solution characteristics. After applying the relocation scheme (27), the adapted finite element grid may include some 'distorted' elements, but these are, in general, elements whose errors are small enough.

The $h$-method in the present work is limited to applications for four-node quadratic and three-node finite elements, and it is based on the grid design problem similar to (22). However, the $h$-method introduced here cannot yield the finite element grid which satisfies the necessary condition in the exact sense. Furthermore, we shall refine grids iteratively instead of
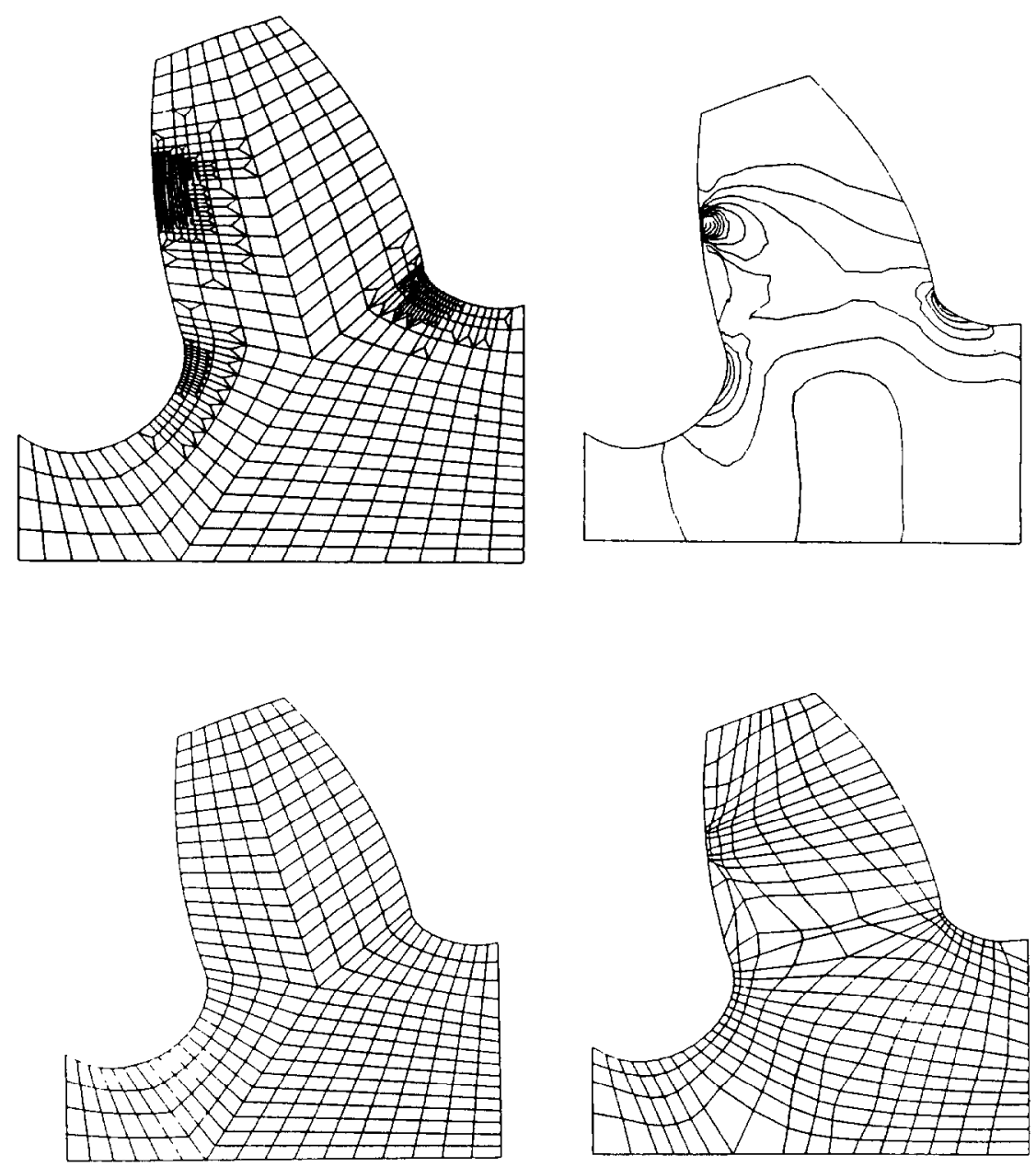

Fig. 9. Adaptive finite element methods ( $r$-and $h$-methods) for a gear tooth. 
determining a necessary degree of refinement according to the so-called error indicator that is reduced under a given level, say, for example, $5 \%$.

To facilitate the latter approach, Babuška and his coworkers introduced several refinements of an appropriate error indicator that provides almost the exact amount of finite element approximation error generated by a given finite element grid. In our case, however, this magnitude of total error is not the issue. The most important thing is the distribution of error and the relative size of error. Once this becomes clear, it is possible to determine which elements must be refined how many times within an allowable number of finite elements. Furthermore, if a quality index, which has a quite similar role with that of the error indicator, is defined by

$$
Q_{1}=\left(\sum_{e} E_{e}^{2}\right)^{1 / 2} / \sqrt{\int_{\Omega} E_{i j k l} \varepsilon_{k l}\left(u_{h}\right) \varepsilon_{i j}\left(u_{h}\right) \mathrm{d} \Omega},
$$

where $u_{h}$ is the finite element solution, it is also possible to indicate the upper bound of the finite element approximation error, although it is not as sharp as the one used by Babuška and his
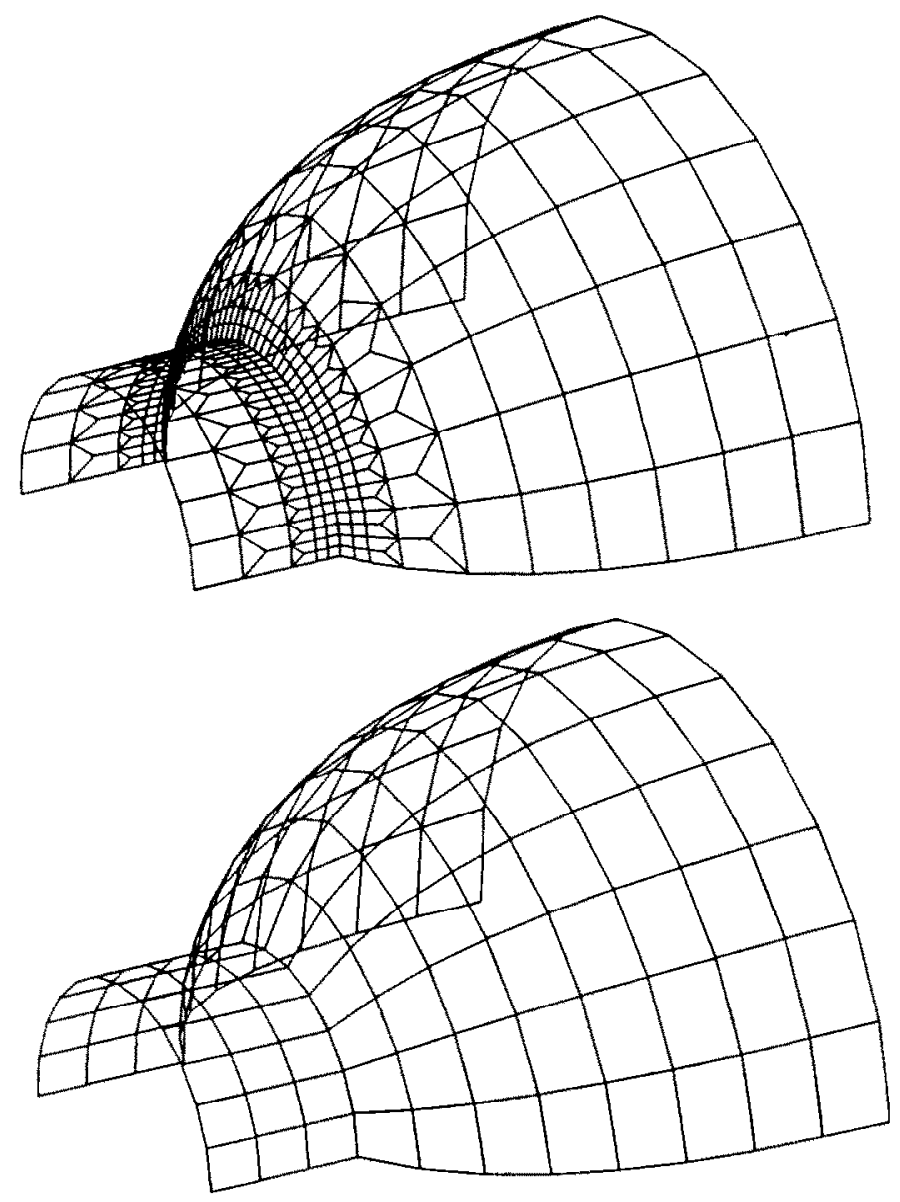

Fig. 10. Application of the $h$-adaptive method for a shell. 
coworkers. It is certain that $Q_{1}$ goes to zero as the finite element approximation error decreases. It is also noted that refinement of finite elements whose error measures are large yields smaller deviation from the average value of error measures than that of the original grid. In this sense, $h$-refinement enforces the necessary condition for optimality of the grid design problem, even though it would not be satisfied in the exact form. If the condition needs to be satisfied exactly, together with substantial reduction of the amount of error, the $r$-and $h$-methods must be applied at the same time.

In the $h$-method, in our work, refinement is performed for finite elements whose error measure is four times larger than the average. If there are no such finite elements, we choose $25 \%-35 \%$ elements of a finite element grid to be refined. These must have larger error measure. We shall repeat this process several times, or until $Q_{1} \leqslant 0.05$ is achieved.

Results for two examples of adaptive methods applied in this paper are given, the one in Fig. 9 for a gear tooth, and in Fig. 10 for a shell structure.

\section{Examples of shape optimization}

The methods described above have been applied to the frequently cited example of the design of a hole in a square plate where tensile forces are evenly distributed along opposite sets of parallel edges. (For an infinite plate the analytical solution is an elliptic hole with an aspect ratio ( 1.5 in this example) proportional to the ratio of stress components at infinity.) The criterion function $F$ for shape optimization is designated to be the von Mises equivalent stress, and the design boundary $\Gamma_{\mathrm{d}}$ is just the hole boundary. Iteration histories of the boundary shape and finite element grids for this problem are shown in Fig. 11. Large oscillations at the early stages quickly fade away and the procedure converges to the elliptical shaped hole. Convergence characteristics are given in Fig. 12. It is clear that for this case the maximum value of the von Mises equivalent stress in the whole domain $\Omega$ is the same as that on the hole boundary $\Gamma_{\mathrm{d}}$. In other words, for the example the design problem (12) is equivalent to the problem defined by (1).

The shape design of a highway road pole is treated as a second example. The problem was solved by Oda and Yamazaki [28]; in their work a hole was created at the place of minimum thickness in order to obtain a fully stressed shape. A similar technique was described in [30] as a way to design the optimal shape. Considering only a half portion of the highway road pole, let us first introduce two design boundaries at the center symmetric line and the right-hand side outside boundary. If the bottom line is allowed to move horizontally, the optimal shape is obtained as shown in Fig. 13, starting from the initial grid specified in the same figure and produced without applying a remeshing scheme during the geometric adaptive iteration. It is also noted that the nodes on the design boundary are moved along the grid direction in the initial finite element grid. Thus, after a number of iterations with an associated large design change, the finite element grid has become quite distorted. As a consequence the final shape obtained from this process has poor quality. In order to obtain an improvement in the design, we set up a modified finite element grid for the restart of the shape design steps. The element connectivities defined in the initial grid are preserved. To obtain the new grid, node locations are modified by applying the $r$-method using the error measures computed at the last previous design stage. The grid shown in Fig. 14 is obtained from this procedure; it is the second initial grid for shape optimization. 

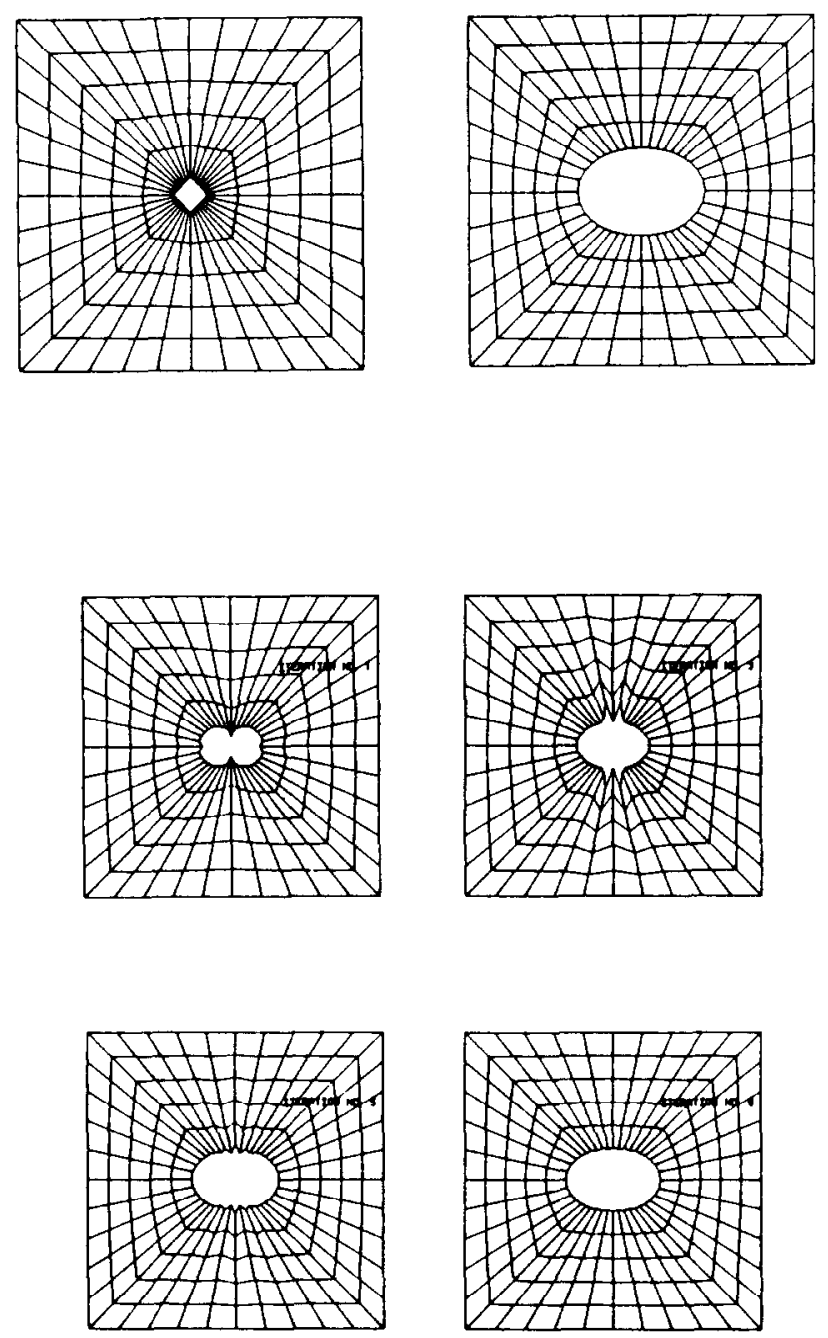

Fig. 11. Design histories of a shape design of a thin elastic plate with a hole.
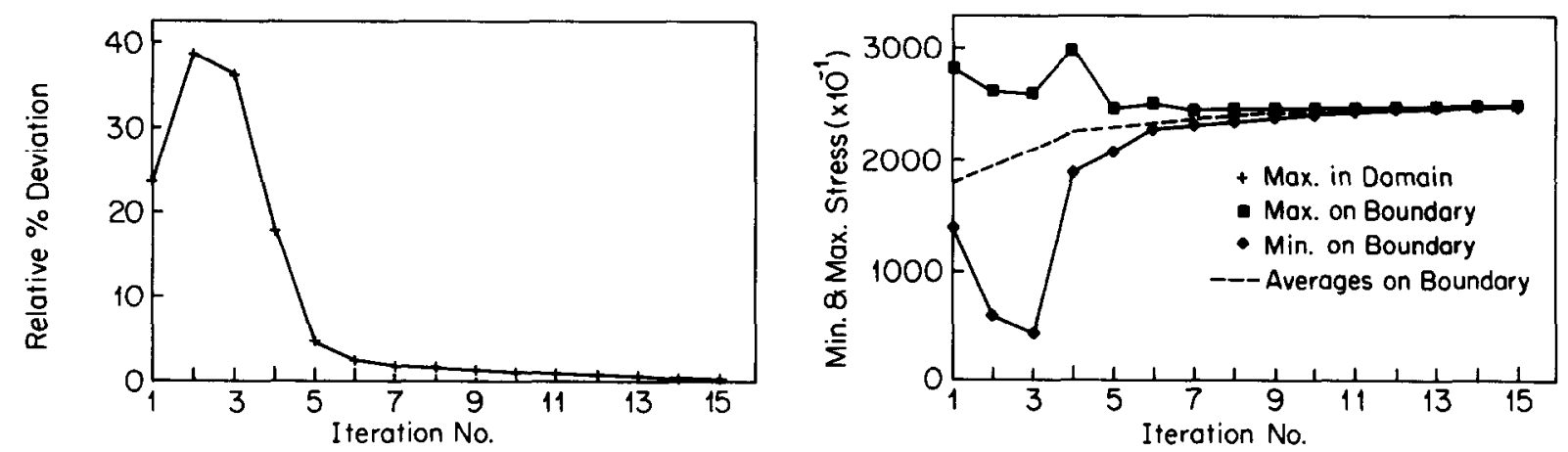

Fig. 12. Convergence history of the geometric adaptive method. 

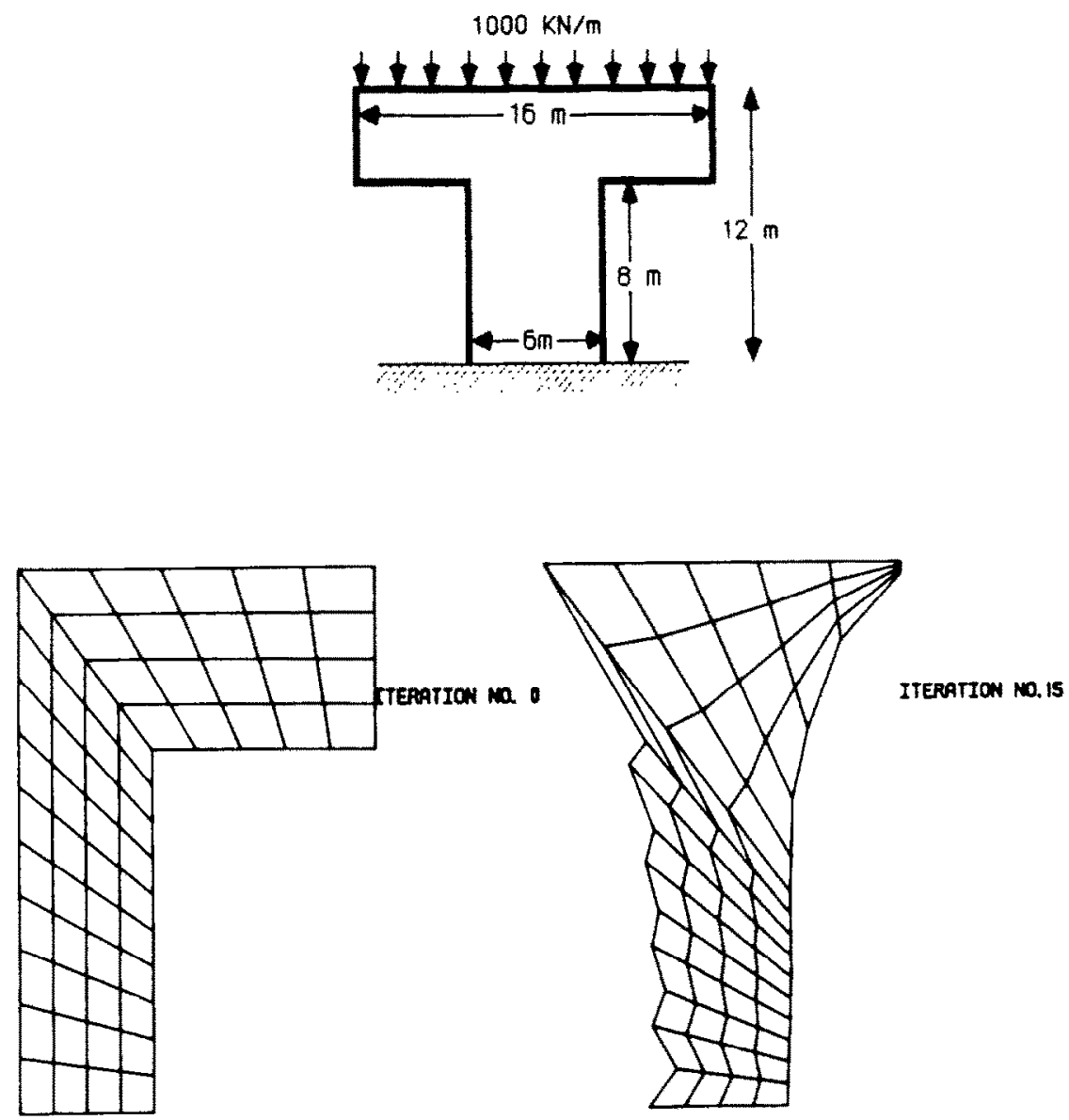

Fig. 13. Road pole shape design problem, initial grid, and result without applying remeshing.
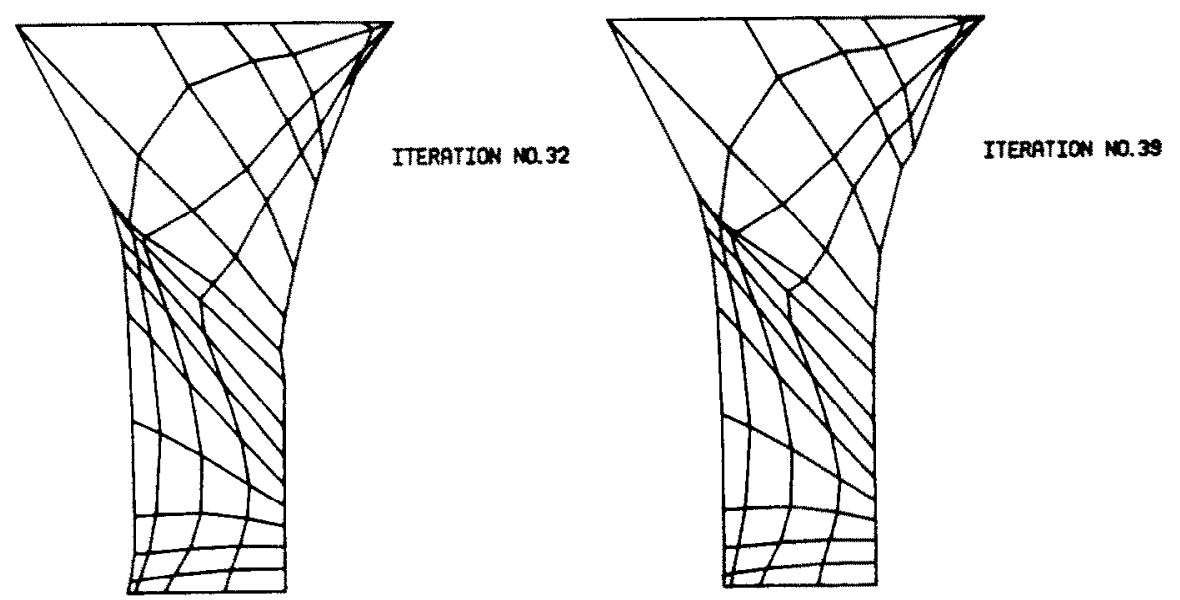

Fig. 14. Remeshed second initial grid and the optimum shape of the road pole. 
After subsequent geometric adaptive steps, the optimal shape is obtained as shown in the figure. It is also noted that if the bottom line is fixed, singular shape design is obtained at the end points of the bottom line. We cannot expect a hole inside of the road pole. Furthermore, if we allow for the possibility that a hole may be generated in the road pole already separated into two parts in the previous design stage, the pole separates again, now into four road poles as shown in Fig. 15. Therefore, if we repeat this process infinitely, many poles are generated so that in the limit the final optimally designed road pole has the configuration of a set of fibers.

Another commonly used example in shape optimization is the fillet design problem. As shown in Fig. 16, if remeshing is not performed during the shape design process, no matter how much the finite element grids are refined, the (optimal) shape produced is not practical. Thus, following the idea for the road pole problem, let us remesh the design boundary to have a smooth boundary. After this step, the finite element grid is reconstructed by applying the $r$-and
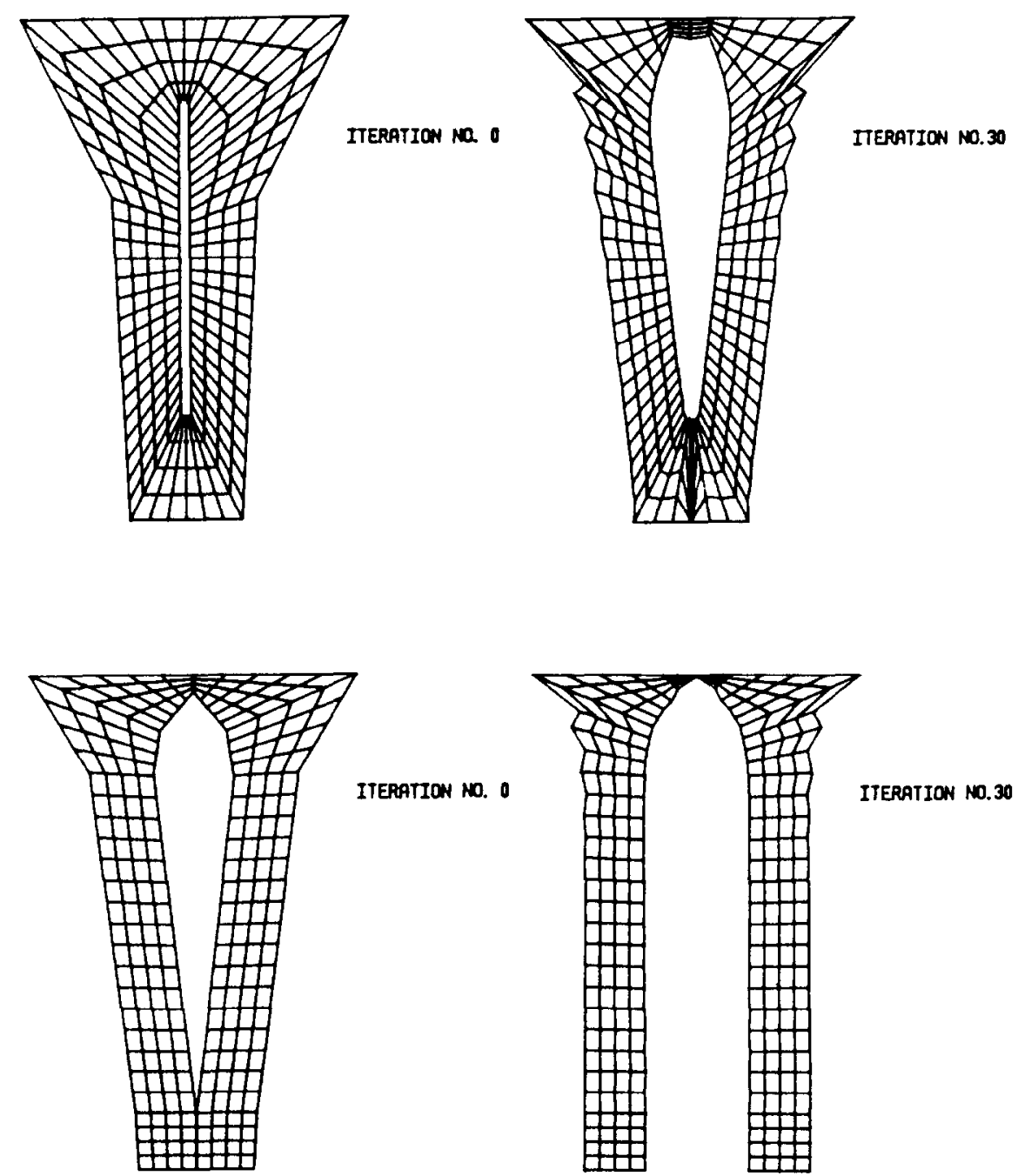

ITERATION NO. 0

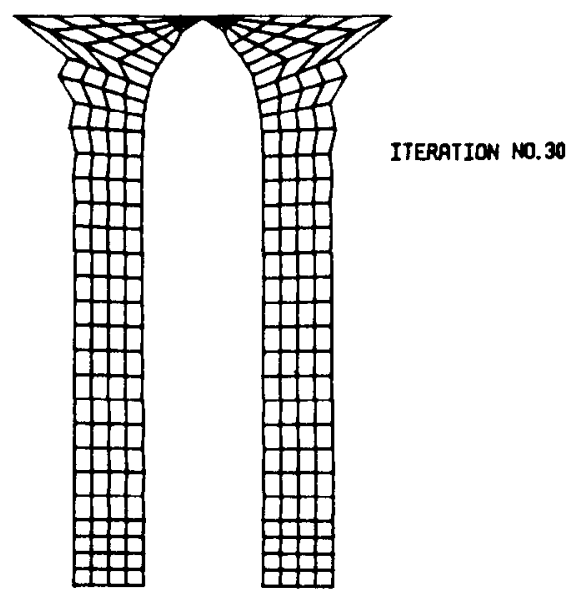

Fig. 15. Continuation of the road pole problem with the possibility of having a hole inside. 

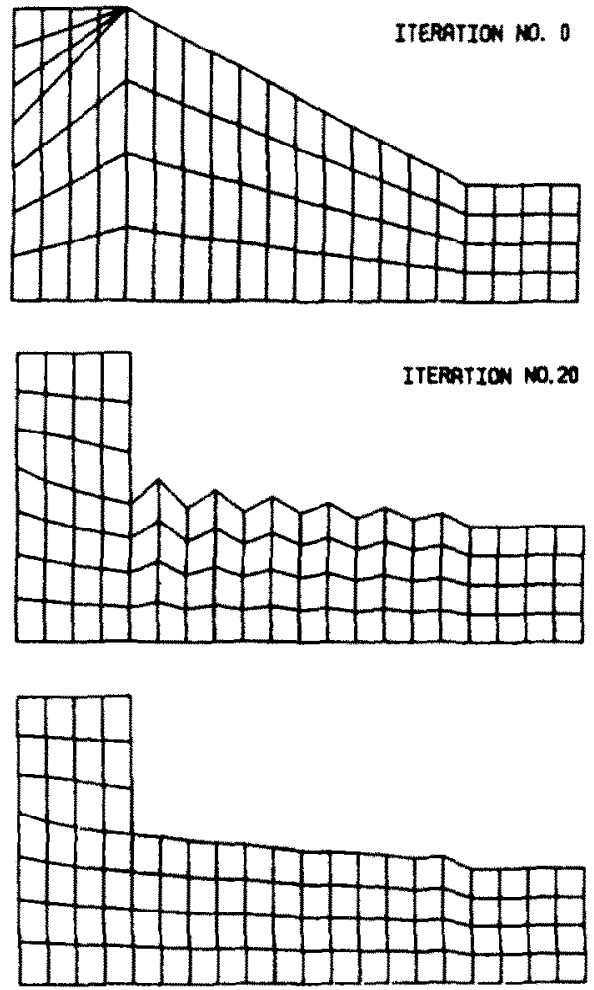

Fig. 16. Initial grid and the optimum shape without remeshing, and the smoothed shape by the least square method.

$h$-methods as shown in Figs. 17 and 18. If the geometric adaptive method is again applied for shape optimization, now based on the second initial grids of Figs. 17 and 18, the optimal shape of the fillet can be obtained without unreasonable oscillations. In this case, it is noted that the value of the maximum von Mises stress in the whole domain is not the same as that on the design boundary, since the right side of the design boundary is also restricted. Thus, the maximum value of the von Mises stress appears outside of the design boundary. In this case, the optimal shape is obtained as shown in Figs. 19. Figure 20 shows a comparison with the photoelastic result obtained by Schnack [29]. The stress fringes from photoelasticity appear to be quite close to those obtained for the 'optimal shape' solution.

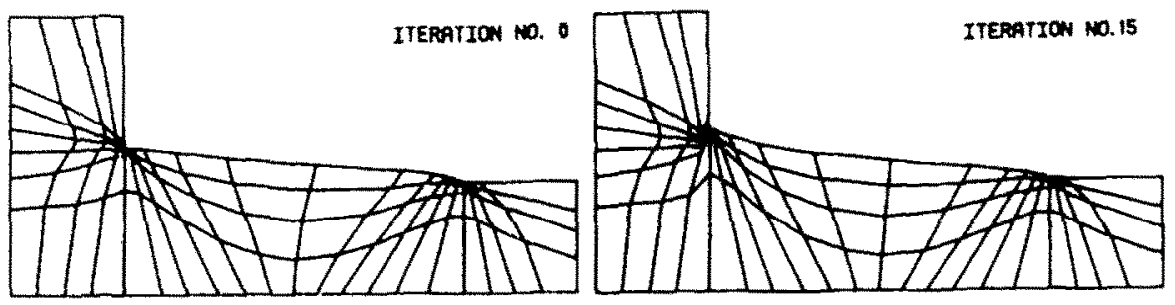

Fig. 17. Remeshed second initial grid by the $r$-method and the optimum shape computed. 

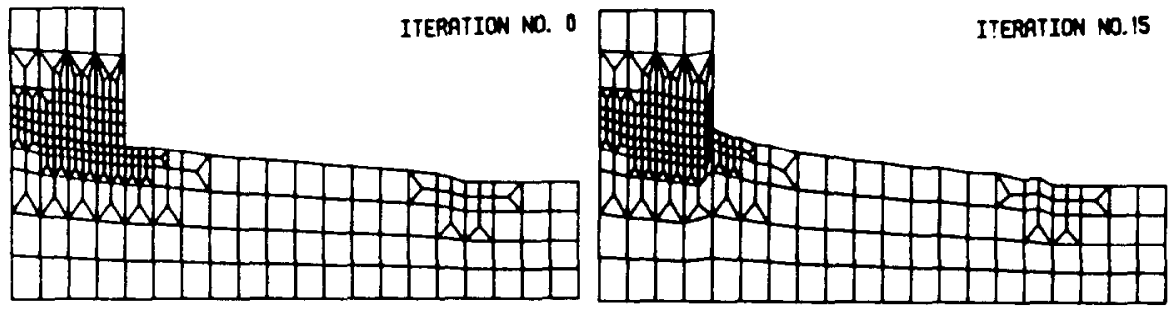

Fig. 18. Remeshed second initial grid by the $h$-method and the optimum shape computed.
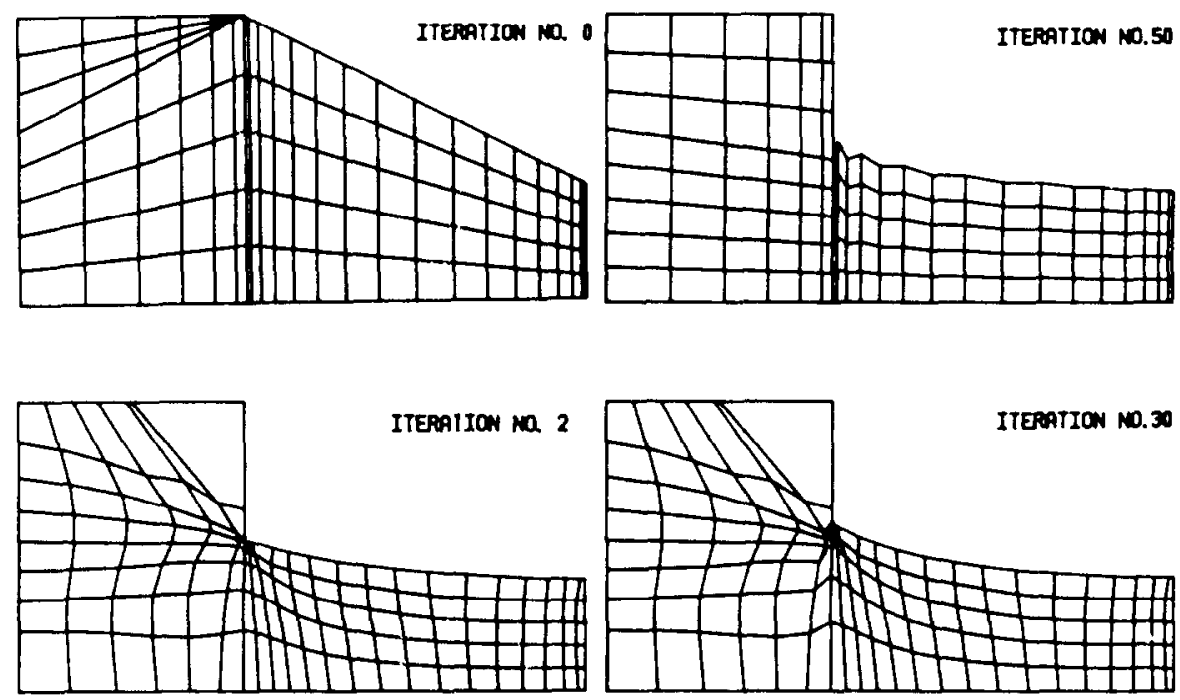

Fig. 19. Shape optimization of a fillet without design restriction along the right edge.
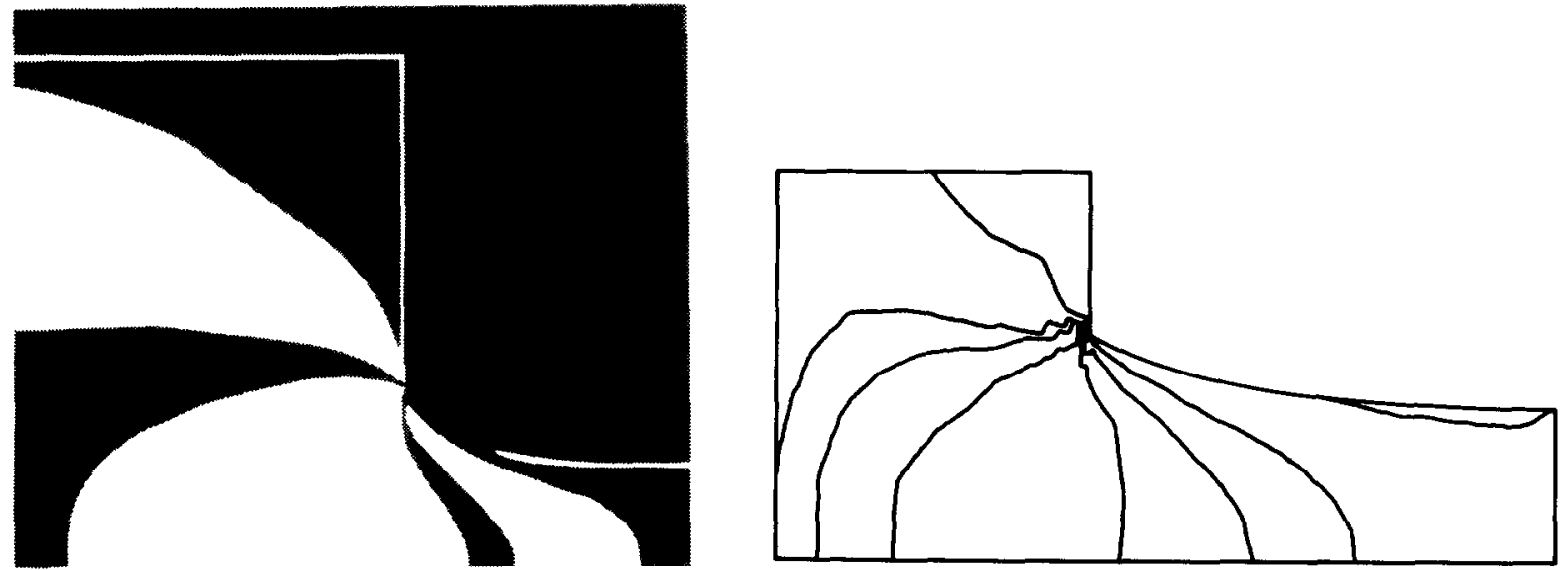

Fig. 20. Fringes obtained by photoelasticity and by the finite element method. 


\section{References}

[1] O.C. Zienkiewicz and J.S. Campbell, Shape optimization and sequential linear programming, in: R.H. Gallagher and O.C. Zienkiewicz, eds., Optimum Structural Design (Wiley, London, 1973) Chapter 7.

[2] C.V. Ramakrishnan and A. Francavilla, Structural shape optimization using penalty functions, J. Structural Mech. 3(4) (1975) 403-432.

[3] V. Tvergaard, On the optimum shape of a fillet in a bar with restrictions, in: Proceedings IUTAM Symposium on Optimization in Structural Design (Springer, Berlin, 1973).

[4] E.S. Kristensen and N.F. Madsen, On the optimum shape of fillets in plates subjected to multiple inplane loading cases, Internat. J. Numer. Meths. Engrg. 10 (1976) 1007-1019.

[5] J.P. Queau and P.H. Trompette, Two-dimensional shape optimal design by finite element method, Internat. J. Numer. Meths. Engrg. 15 (1980) 1603-1612.

[6] J. Oda and K. Yamazaki, A procedure to obtain a fully stressed shape of elastic continuum, Internat. J. Numer. Meths. Engrg. 15 (1980) 1095-1105.

[7] N.V. Banichuk, Problems and Methods of Optimal Structural Design, Translated by V. Komkov and E.J. Haug (Plenum Press, New York, 1983).

[8] K. Dems, Multiparameter shape optimization of elastic bars in torsion, Internat. J. Numer. Meths. Engrg. 15 (1980) 1517-1539.

[9] K. Dems and Z. Mroz, Optimal shape design of multicomposite structures, J. Structural Mech. 8(3) (1980) $309-329$.

[10] K. Dems and Z. Mroz, Variational approach by means of adjoint systems to structural optimization and sensitivity analysis-I, Internat. J. Solids and Structures 19(8) (1983) 677-692.

[11] K. Dems and Z. Mroz, Variational approach by means of adjoint systems to structural optimization and sensitivity analysis-II, Internat. J. Solids and Structures 20(6) (1984) 527-552.

[12] K.K. Choi and E.J. Haug, Shape design sensitivity analysis of elastic structures, J. Structural Mech. 11(2) (1983) 231-269.

[13] M.S. Na, N. Kikuchi and J.E. Taylor, Optimal modification of shape for two-dimensional elastic bodies, J. Structural Mech. 11(1) (1983) 111-135.

[14] N. Olhoff and J.E. Taylor, On optimal structural remodeling, J. Optim. Theory Appl. 27(4) (1979) 571-582.

[15] N. Kikuchi, Adaptive grid-design methods for finite element methods, Comput. Meths. Appl. Mech. Engrg. 55 (1986) 129-159.

[16] I. Babuška and M.R. Door, Error estimates for the combined $h$ and $p$ version of the finite element method, Numer. Math. 25 (1981) 257-277.

[17] I. Babuška and W.C. Rheinboldt, Error estimates for adaptive finite computations, SIAM J. Numer. Anal. 15 (1978) 736-754.

[18] I. Babuška and W.C. Rheinboldt, Reliable error estimation and mesh adaptation for the finite element method, in: J.T. Oden, ed., Computational Methods in Nonlinear Mechanics (North-Holland, Amsterdam, 1980) 67-109.

[19] I. Babuška and B. Szabó, On the rate of convergence of the finite element method, Internat. J. Numer. Meths. Engrg. 18 (1982) 323-341.

[20] I. Babuška, A. Miller and M. Vogelius, Adaptive methods and error cstimation for elliptic problems of structural mechanics, in: I. Babuška, J. Chandra and J.E. Flaherty, eds., Adaptive Computational Methods of Partial Differential Equations (SIAM, Philadelphia, PA, 1983) 57-73.

[21] M.S. Shephard, R.H. Gallagher and J.F. Abel, The synthesis of near optimum finite element meshes with interactive computer graphics, Internat. J. Numer. Meths. Engrg. 15 (1980) 1021-1039.

[22] O.C. Zienkiewicz, J.P. Gago and D.W. Kelly, The hicrarchical concept in finite element analysis, Comput. \& Structures 16 (1983) 53-65.

[23] M.S. Shephard, ed., Finite Element Grid Optimization PVP-38. (ASME, New York, 1979).

[24] I. Babuška, J. Chandra and J.E. Flaherty, eds., Adaptive Computational Methods for Partial Differential Equations (SIAM, Philadelphia, PA, 1983).

[25] E.R. Oliveira de Arantes, I. Babuška, O.C. Zienkiewicz and J.P. Gago, eds., Proceedings of International Conference on Accuracy Estimates and Adaptive Refinements in Finite Elements Computation, Universidade Técnica de Lisboa, Lisbon, 1984. 
[26] K.Y. Chung, Shape optimization and free boundary problems with grid adaptation, Dissertation at The University of Michigan, Ann Arbor, MI, 1985.

[27] A.M. Winslow, Numerical solution of quasilinear Poisson equation in a nonuniform triangular mesh, J. Comput. Phys. 2 (1967) 149-172.

[28] J. Oda and K. Yamazaki, On a technique to obtain an optimum strength shape of an axisymmetric body by the finite element method, Bull. JSME 20(150) (1977) 1524-1532.

[29] E. Schnack, An optimization procedure for stress concentration by finite element techniques, Internat. J. Numer. Meths. Engrg. 14 (1979) 115-124.

[30] M.P. Rossow and I.E. Taylor, An optimal structural design algorithm using optimality criteria, Society Engineering Science, 13th Annual Meeting, Hampton, VA, 1976. 